\title{
Espectrocolorimetria de variedades de caulim da Jazida Ipixuna, no Estado do Pará
}

\author{
Spectrocolorimetry of kaolin varieties \\ from Ipixuna ore, in Pará State
}

\author{
Sydney Sabedot \\ Professor, Doutor do Mestrado \\ em Avaliação de Impactos Ambientais \\ em Mineração, do Unilasalle \\ sabedot@unilasalle.edu.br

\section{Carlos Otávio Petter \\ Professor, Doutor do PPGEM, da UFRGS cpetter@ufrgs.br} \\ Carlos Hoffmann Sampaio \\ Professor, Doutor do PPGEM, da UFRGS \\ sampaio@ufrgs.br
}

\section{Resumo}

Variedades de minério de caulim amostradas em uma jazida sedimentar no Estado do Pará foram beneficiadas em planta-piloto para estudos de espectrocolorimetria que possibilitassem a criação de uma ferramenta de apoio operacional à lavra e ao beneficiamento. Jazidas como esta contêm blocos com variações texturais e mineralógicas, as quais definem os blocos de minério com potencial para beneficiamento. A tomada de decisão depende, inicialmente, da análise subjetiva da cor do minério: blocos com cores avermelhadas são impróprios; com cores esbranquiçadas são apropriados. A cor do minério depende da paragênese mineral contaminante. Quinze amostras representativas de cinco tipos de caulins mais comuns na jazida, definidos visualmente pela cor do minério, foram analisadas sob distintos parâmetros. As curvas de reflectância do produto desareado das amostras definiram sete grupos espectrocolorimétricos, sendo quatro próprios para gerarem uma matériaprima final com qualidade para o mercado. Esse estudo apresenta as análises e os resultados para cada grupo e o que eles representam como ferramenta de suporte à lavra e ao beneficiamento. Também apresenta algumas considerações quanto à blendagem entre os grupos de caulim.

Palavras-chave: Minério de caulim, espectrocolorimetria, curvas de reflectância.

\section{Abstract}

Varieties of kaolin ore were sampled from a sedimentary deposit in Pará state and processed in a pilot plant for spectrocolorimetry studies. The aim was to provide a tool for the mining and processing operations. Ores like that contain textural and mineralogical variations that define whether the ore blocks may or may not be processed. The decision making depends, first, on the ore color subjective analysis: reddish blocks are inappropriate, but the whitish ones are appropriate. The ore color depends on the mineral paragenesis contaminant. Fifteen representative samples of five varieties of kaolin were analyzed under different parameters. These samples were visually defined by the ore color. The reflectance curves of the sandless product defined seven spectrocolorimetric groups, four of which were appropriate for generating a quality product for the market. This study presents both the analyses and the results for each group, what they represent as a tool to support the mining and processing operations, and some considerations about the blending among the kaolin groups.

Keywords: Kaolin ore, spectrocolorimetry, reflectance curves. 


\section{Introdução}

Caulim é um termo comercial usado para denominar uma argila branca composta essencialmente pelo mineral caulinita (Bloodworth et al., 1993). $\mathrm{O}$ minério run-of-mine (ROM) pode conter outros minerais sob a forma de impurezas, desde traços a até $50 \%$ em volume, consistindo, em geral, de quartzo, micas, feldspatos, óxidos de ferro e de titânio, entre outros. (Luz, et al., 2005). A presença desses minerais afeta o beneficiamento do ROM e o uso da matéria-prima final, dependendo de seus teores na mesma. $O$ caulim pode ser aplicado em diversos segmentos industriais, tais como tintas, cerâmicas, refratários, catalisadores, louças de mesa, peças sanitárias, cimento branco, borrachas, plásticos, adesivos, vidros, cosméticos e pesticidas; mas a indústria de papel (cobertura e enchimento) é o segmento mais importante, representando cerca de $45 \%$ do mercado mundial. O Brasil responde por cerca de $7 \%$ da produção mundial. Cerca de 97\% das reservas medidas e indicadas brasileiras encontram-se nos estados do Pará, Amapá e Amazonas (Sena \&

\section{Materiais e métodos}

A heterogeneidade da jazida Ipixuna se configura, de acordo com o pessoal operacional, com cinco tipos de caulim ROM, considerando suas características mineralógicas, texturais e de cor de sedimento, que varia, tanto na distribuição horizontal, como na vertical. Os tipos são definidos visualmente e, sob esta condição, foi solicitado para o pessoal operacional que coletasse três amostras de cada tipo. Aproveitando as diversas frentes de lavra, as amostras foram coletadas em áreas e profundidades diferentes, permitindo um estudo espacialmente amplo.

Quinze amostras, cada uma com cerca de uma tonelada, foram beneficiadas em planta-piloto, na empresa, para a obtenção de produtos e rejeitos compatíveis com o processo industrial. Os processos de beneficiamento, partindo do ROM, foram: dispersão, desareamento (que gerou o produto desareado), centrifugação (idem, centrifugado), separação magnética (idem, não magnético), branqueamento químico (idem, branqueado),
Mártires, 2008; Roskill, 2006; Murray, 2000; Prasada et al., 1991).

Amostras coletadas na Mina Ipixuna, um significativo depósito sedimentar no município homônimo, no Estado do Pará, com coordenadas geográficas aproximadas de $02^{\circ} 23^{\prime} \mathrm{S}$ e $47^{\circ} 45^{\prime} \mathrm{W}$, explorado pela empresa Vale, serviram de base para o estudo. A jazida é heterogênea quanto às características físicas, químicas e mineralógicas na distribuição horizontal e vertical dos sedimentos, não raras vezes de forma abrupta. As variações têm relação com os eventos sin ou pós-deposicionais. Um exemplo é a percolação de água rica em ferro, que altera a cor e a mineralogia original em parte dos sedimentos. Blocos assim constituídos podem afetar as operações de lavra e beneficiamento. A decisão do operador para o aproveitamento depende da sua percepção visual para a cor e textura do bloco, isto é, com base em critérios subjetivos. E o beneficiamento implica o uso e a dosagem de produtos químicos, bem como a definição de parâmetros operacionais durante as sucessivas etapas, com efeito direto nos custos e na qualidade do produto final. Outro aspecto importante, e que também afeta os itens anteriormente citados, é a blendagem de tipos diferentes de minério ROM durante o beneficiamento. A mineralogia dos contaminantes e suas concentrações podem exigir parâmetros operacionais distintos no beneficiamento. Nesse estudo, caulins ROM com cores avermelhadas produziram produtos finais com diferentes alvuras e teores de óxidos de ferro e titânio, determinando que um grupo ROM fosse de qualidade e outro não. Sob esse contexto, esse estudo objetiva estabelecer uma ferramenta de apoio operacional à lavra e ao beneficiamento da jazida Ipixuna, tendo, como base, o espectro de reflectância de produtos desareados, da primeira etapa de beneficiamento do ROM. Essa ferramenta pode substituir o procedimento que implica o beneficiamento total da amostra em laboratório, possibilitando decisões mais objetivas, rápidas e eficazes, tanto na demanda de blocos para o beneficiamento, quanto para a blendagem de variedades de minério ROM. filtragem e secagem (idem, final). De cada fase, foram retiradas alíquotas de produtos que fundamentaram esse estudo.

Diferente de um trabalho anterior, realizado em jazida similar no Estado do Amapá (Sabedot et al., 1998), cujo espectro de referência foi o do minério ROM, nesse estudo, partiu-se do espectro do produto desareado, considerado como um avanço em relação ao estudo anterior, devido a dois motivos: o primeiro relacionase à incorporação de contaminantes grossos e colorantes do ROM na moagem da amostra.

Esse procedimento gera um espectro ROM que pode ser interpretado como minério não-econômico, porque os contaminantes colorantes grossos interferem na forma do espectro. No entanto, o produto desareado, sem aquela fração, origina um espectro diferente, cuja forma permite avaliar melhor a condição do minério, isto é, se pode ou não ter aproveitamento econômico. O segundo motivo é a presença de quartzo no ROM, que é retirado no processo de desareamento. O teor variável de quartzo também pode gerar espectros diferenciados e influir na forma do espectro do ROM. Sua diminuição no produto desareado gera um espectro menos susceptível à influência daquele mineral e uma condição mais confiável para a definição do aproveitamento do bloco.

As alíquotas de produtos e rejeitos do beneficiamento em planta-piloto foram remetidas ao Laboratório de Processamento Mineral, da UFRGS, onde foram pulverizadas sob procedimentos e equipamentos idênticos aos das operações de laboratório na mina.

As medidas de reflectância das alíquotas pulverizadas foram realizadas em um espectrofotômetro da marca Minolta, modelo 2600d, similar ao utilizado no laboratório da mina. Considerou-se a faixa de comprimento de onda entre 360 e $740 \mathrm{~nm}$.

Todas as alíquotas derivadas dos processos de beneficiamento das 15 amostras foram analisadas por fluorescência de raios $x$, no Instituto de Geociências da UFRGS. 


\section{Resultados}

Os resultados da espectrofotometria foram tratados em planilha Excel, a qual possibilitou a definição do conjunto de curvas para os produtos do beneficiamento: desareado, centrifugado, não-magnético, branqueado (úmido) e final (seco). Ainda que o interesse estivesse centrado no produto desareado,

Figura 1

Espectros do produto desareado dos sete grupos colorimétricos de caulim.

Figura 2

Espectros do Grupo I.

Figura 3

Espectros do Grupo II.

Figura 4

Espectros do Grupo III.

Figura 5

Espectros do Grupo IV.

Figura 6

Espectros do Grupo V.

Figura 7

Espectros do Grupo VI.

Figura 8

Espectros do Grupo VII.

As quinze amostras ficaram assim distribuídas: quatro no Grupo I, duas as demais curvas ajudaram a compreender melhor o desempenho do minério nas diversas etapas do beneficiamento, possibilitando definir se aquele espectro (desareado) suportaria a condição para gerar um produto final de qualidade.

A análise dos conjuntos de espectros de reflectância das 15 amostras
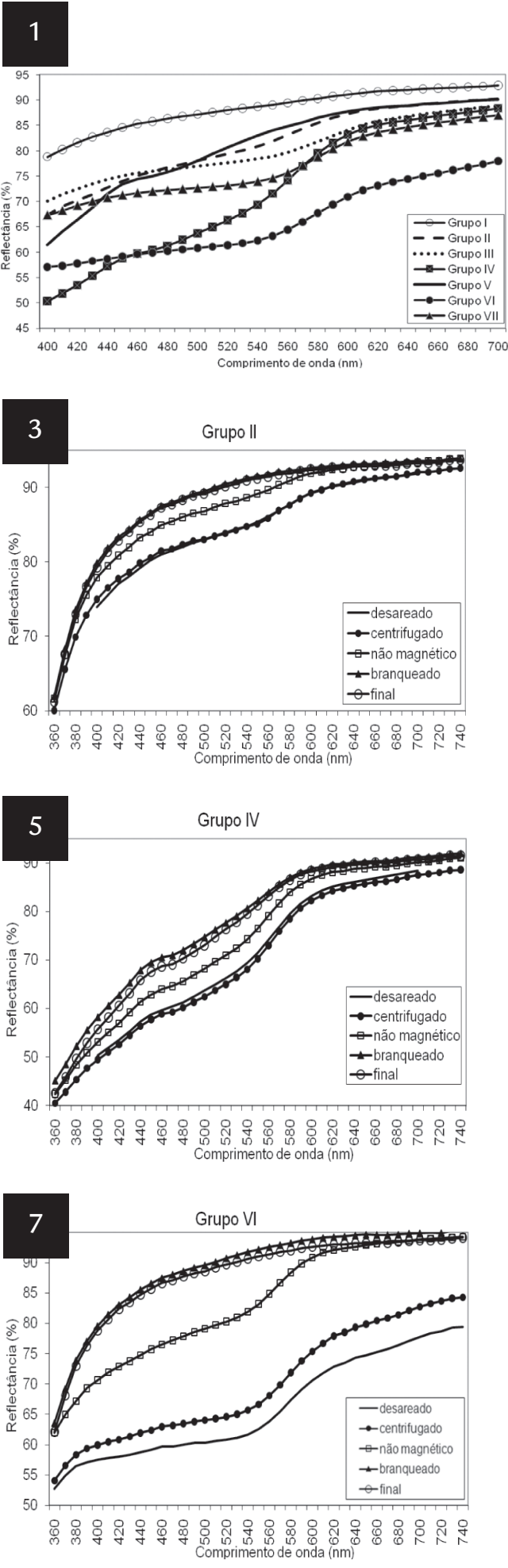

no Grupo II, quatro no Grupo III, uma no Grupo IV, uma no Grupo V, uma no possibilitou uma classificação em sete grupos de caulim, que, nesse contexto, podem ser considerados como sete variedades colorimétricas da jazida Ipixuna. A Figura 1 mostra os espectros do produto desareado dos grupos. As Figuras 2 a 8 mostram a evolução dos espectros com o beneficiamento, em cada grupo.
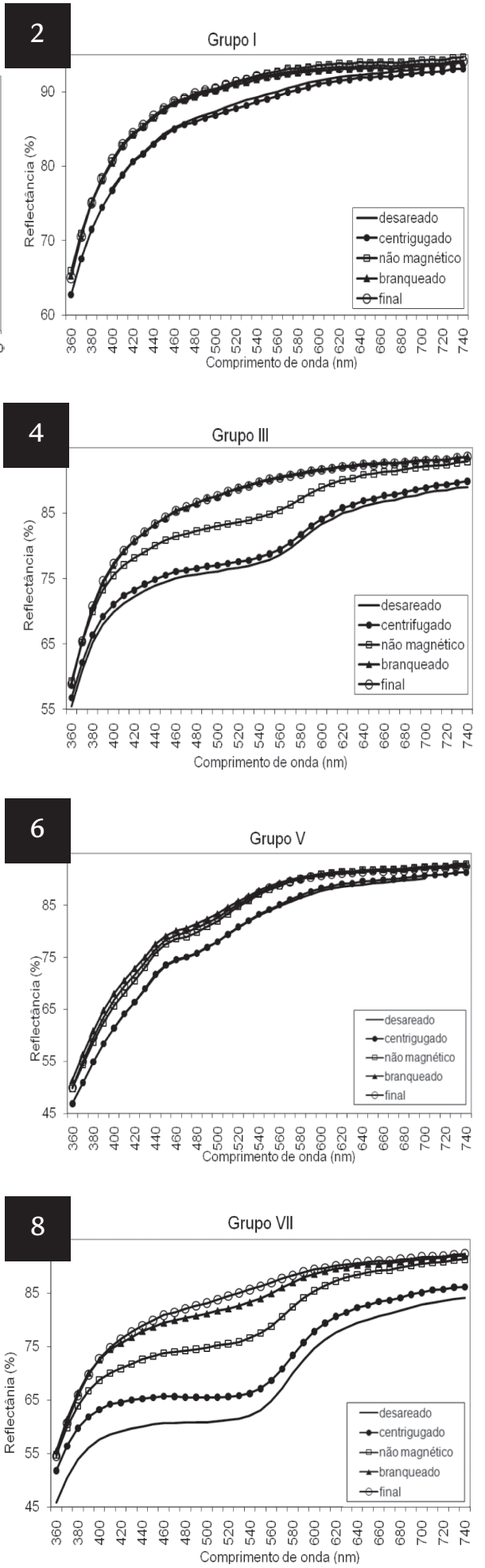

Grupo VI e duas no Grupo VII. A Tabela 1 mostra os valores médios de Alvura 
ISO, Yellowness, e teores dos óxidos de Fe e Ti para os produtos desareado e fi-

\begin{tabular}{|c|c|c|c|c|c|}
\hline Grupo & Produto & Alvura (\%) & Yellowness (\%) & $\mathrm{Fe}_{2} \mathrm{O}_{3}(\%)$ & $\mathrm{TiO}_{2}(\%)$ \\
\hline \multirow{2}{*}{1} & $\mathrm{D}$ & 85,01 & 7,4 & 0,3 & 0,82 \\
\hline & $\mathrm{F}$ & 89,48 & 5,23 & 0,29 & 0,67 \\
\hline \multirow{2}{*}{ II } & $\mathrm{D}$ & 77,5 & 11,83 & 0,67 & 1,35 \\
\hline & $\mathrm{F}$ & 87,0 & 7,13 & 0,64 & 1,2 \\
\hline \multirow{2}{*}{ III } & $\mathrm{D}$ & 73,24 & 12,19 & 0,9 & 1,52 \\
\hline & $\mathrm{F}$ & 86,12 & 7,36 & 0,54 & 1,04 \\
\hline \multirow{2}{*}{ IV } & $\mathrm{D}$ & 59,03 & 29,39 & 1,17 & 0,98 \\
\hline & $\mathrm{F}$ & 70,4 & 21,64 & 0,96 & 0,86 \\
\hline \multirow{2}{*}{ V } & $\mathrm{D}$ & 73,38 & 16,38 & 1,07 & 1,48 \\
\hline & $\mathrm{F}$ & 80,35 & 12,95 & 0,98 & 1,23 \\
\hline \multirow{2}{*}{$\mathrm{VI}$} & $\mathrm{D}$ & 59,39 & 15,49 & 2,82 & 0,82 \\
\hline & $\mathrm{F}$ & 87,38 & 6,81 & 0,35 & 0,75 \\
\hline \multirow{2}{*}{ VII } & $\mathrm{D}$ & 69,01 & 12,88 & 1,14 & 1,61 \\
\hline & $\mathrm{F}$ & 83,41 & 8,41 & 1,11 & 1,81 \\
\hline
\end{tabular}

Observação:D - produto desareado; F - produto final.

\section{Discussão}

Para o Grupo I, as curvas dos produtos desareado ao final têm formas similares e convexas. As curvas dos produtos não-magnético, branqueado e final estão praticamente superpostas, indicando que o branqueamento pouco acrescentou em termos de qualidade. O desareado inicia com alvura elevada e o beneficiamento incrementa poucos pontos de alvura devido aos baixos teores de $\mathrm{Fe}_{2} \mathrm{O}_{3}$ e $\mathrm{TiO}_{2}$, sendo que, para o ferro, os teores, praticamente, se mantiveram e, para o titânio, houve uma pequena redução, com reflexos na diminuição do yellowness. Esse conjunto de curvas (Figura 2) indica um caulim ROM pouco contaminado, cujo beneficiamento exige parâmetros operacionais mais brandos e gera um produto final de excelente qualidade. Essas características sugerem que o beneficiamento dessa variedade seria mais eficaz sem blendagem com as demais variedades.

O Grupo II apresenta a curva desareado com uma leve concavidade em torno do comprimento de onda $550 \mathrm{~nm}$ e em um patamar de reflectância mais baixo, comparado com o da curva desareado do Grupo I, devido aos teores mais elevados de $\mathrm{Fe}_{2} \mathrm{O}_{3}$ e $\mathrm{TiO}_{2}$. Apesar da pouca redução do ferro e titânio entre os produtos desareado e final, os processos levam a um excelente ganho de alvura e a uma boa redução do yellowness; há eficácia no branqueamento, que remove o ferro, evidenciado na comparação entre os formatos das curvas não-magnético e branqueado, na Figura 3. O conjunto de curvas, nesse grupo, também caracteriza um caulim ROM adequado para o beneficiamento e com possibilidade de gerar um produto final de boa qualidade.

As principais características do Grupo III são: curvas desareado, centrifugado e não-magnético com concavidades acentuadas em torno do comprimento de onda $550 \mathrm{~nm}$; teores elevados de $\mathrm{Fe}_{2} \mathrm{O}_{3}$ e $\mathrm{TiO}_{2}$ no desareado, reduzidos com o beneficiamento, condicionando, para o produto final, incremento no valor de alvura e redução no de yellowness. A alvura do produto final e a seqüência de curvas (Figura 4) indicam que essa variedade de caulim ROM também pode ser submetida ao beneficiamento para obtenção de uma matéria-prima final aceitável no mercado. Os Grupos II e III têm comportamentos de curva e resultados similares, o que permitiria blendagem entre essas variedades no beneficiamento.

O Grupo IV mostra um conjunto de curvas similares quanto à forma, do desareado ao final. Ainda que os processos proporcionem aumento de alvura e redução do yellowness, os valores finais são incompatíveis com a qualidade exigida no mercado. Isso se deve aos elevados teores de $\mathrm{Fe}_{2} \mathrm{O}_{3}$ e $\mathrm{TiO}_{2}$, cujos minerais contaminantes que os contêm não são removidos com eficácia no beneficiamento. A seqüência de curvas que caracteriza o Grupo IV (Figura 5) indica uma variedade de caulim ROM que não deve ser beneficiada na Mina Ipixuna, porque não atinge a alvura esperada e gera um produto final de baixa qualidade.

A curva desareado do Grupo V (Figura 6) é semelhante à mesma curva do Grupo IV, mas há diferenças nos resultados: no Grupo V, os valores de reflectância, em geral, iniciam mais altos; os nal, em cada grupo.

Tabela 1

Valores colorimétricos e químicos dos grupos de caulim.

ganhos de alvura e as reduções de yellowness são pouco significativos com os processos de beneficiamento, o que pode ser constatado com a proximidade entre as curvas, nas figuras; a convexidade do desareado inicia na proximidade do comprimento de onda $450 \mathrm{~nm}$, porém em nível de reflectância mais alto (em torno de $73 \%$, enquanto que no Grupo IV inicia em torno de 57\%); os teores de $\mathrm{Fe}_{2} \mathrm{O}_{3}$ no desareado dos dois grupos são semelhantes, mas o teor de $\mathrm{TiO}_{2}$ no mesmo produto do Grupo V é cerca de 50\% maior que o do Grupo IV. A evolução e o formato das curvas do Grupo V também indicam uma variedade de ROM que não deve ser beneficiada, pelo mesmo motivo citado no Grupo IV.

As curvas do Grupo VI apresentam uma evolução interessante com o beneficiamento. O desareado apresenta reflectâncias baixas nos comprimentos de onda mais baixos, não tem inflexão nas proximidades do comprimento de onda $450 \mathrm{~nm}$, tem elevadíssimo teor de $\mathrm{Fe}_{2} \mathrm{O}_{3}$, que determina um baixíssimo valor para a alvura e um altíssimo valor para o yellowness e um valor intermediário para o teor de $\mathrm{TiO}_{2}$. Com a centrifugação quase não há incremento de qualidade. Porém a separação magnética e o branqueamento provocam consideráveis ganhos de qualidade na alvura e no yellowness. Os teores de $\mathrm{Fe}_{2} \mathrm{O}_{3}$ reduzem acentuadamente naqueles processos, diferentemente dos de $\mathrm{TiO}_{2}$, que pouco reduzem. Visualmente, as cores das variedades de caulim classificadas como Grupos IV, V e VI são semelhantes, o que poderia decretar, na análise visual, o descarte de variedades 
classificadas como Grupo VI. No entanto, os dados obtidos, nesse estudo, indicam que o caulim ROM, cujas curvas se assemelhem às do Grupo VI (Figura 7), deve ser enviado ao beneficiamento, porque proporciona um produto final com boa alvura para o mercado. As características dessa variedade sugerem que a mesma não deve ser blendada com as demais, devido à alta concentração inicial de contaminantes colorantes, ainda que os processos permitam um produto final de boa qualidade. Resultados como os obtidos, nessa variedade ROM, mostram

\section{Conclusões}

Esse estudo possibilitou concluir, inicialmente, que há diferenças quanto à classificação de minério de caulim ROM através do método visual, que considera a percepção subjetiva da cor pelo pessoal operacional, e do método espectrocolorimétrico, que considera a curva de reflectância do produto desareado: pelo primeiro, definem-se cinco tipos de caulins; pelo segundo, sete variedades (ou grupos). Ainda que a análise da curva também seja de modo subjetivo, ela estabelece um critério mais confiável, tendo como base parâmetros físicos objetivos da amostra.

Considerando as características dos sete grupos, pode-se concluir que as variedades de caulim ROM dos grupos IV e V não constituem minério, porque não há alteração no formato das curvas inicial (desareado) e final (final). Os resultados paralelos que auxiliam e também fundamentam o estudo mostram que os parâmetros colorimétricos do produto final estão inadequados e os teores de $\mathrm{Fe}_{2} \mathrm{O}_{3}$ e $\mathrm{TiO}_{2}$ estão altos para uma matéria-prima final de qualidade. As variedades que caracterizam o Grupo VII também não podem ser consideradas minérios, porque os teores de $\mathrm{Fe}_{2} \mathrm{O}_{3}$ e $\mathrm{TiO}_{2}$ não apresentam suficiente dominuição no produto final. Ainda que a curva desareado do Grupo VII seja semelhante ao desareado do Grupo VI, o fator principal no Grupo VII é a presença excessiva de quartzo,

\section{Agradecimentos}

Os autores agradecem à empresa Vale, por disponibilizar amostras e recursos financeiros para os estudos, que ainda continuam. Agradecimentos especiais aos engenheiros químicos Paulo Sérgio que um estudo mais completo envolvendo blendagem $\mathrm{x}$ parâmetros operacionais $\mathrm{x}$ rendimento (alvura) poderia indicar rotas alternativas de beneficiamento mais eficazes na mina.

O desareado do Grupo VII (Figura 8) tem uma curva semelhante ao mesmo produto do Grupo VI, porém mais horizontalizada entre os comprimentos de onda 450 e $550 \mathrm{~nm}$. Situação semelhante ocorre com a curva centrifugado. Os processos de beneficiamento promovem ganhos consideráveis em termos de alvura e yellowness, mas o pro-

que lhe confere uma textura diferente da dos demais grupos. Além disso, a cor do ROM desse grupo é cinza-clara, enquanto que a cor do ROM do Grupo VI é avermelhada. A curva desareado do Grupo I caracteriza uma variedade ROM de excelente qualidade e que, dado às características analisadas, recomendar-se-ia um beneficiamento sem blendagem com outras variedades, devido à boa possibilidade de gerar uma matéria-prima de excelente qualidade, com a aplicação de parâmetros operacionais mais brandos. As variedades dos Grupos II e III podem ser blendadas no beneficiamento. Ainda que tais variedades apresentem teores variáveis de $\mathrm{Fe}_{2} \mathrm{O}_{3}$ e $\mathrm{TiO}_{2}$, a remoção dos minerais contaminantes é eficaz nos consecutivos processos. É provável que a paragênese dos minerais contaminantes seja semelhante nos dois grupos, decorrendo daí semelhanças quanto à eficácia dos processos na retirada dos contaminantes e condicionando valores de alvura semelhantes no produto final, em ambos os grupos. O caulim do Grupo VI tem características interessantes: visualmente, tem cor semelhante aos caulins dos Grupos IV e V; os valores de alvura do desareado são muito baixos; o teor de $\mathrm{Fe}_{2} \mathrm{O}_{3}$ no desareado é cerca de 2,5 vezes maior que o teor do óxido no mesmo produto dos Grupos IV e V e cerca de 10 vezes maior que o teor do óxido no desareado do Grupo I. Essas características, combina-

Criscuolo e Orlando Ferreira e ao geólogo Giorgio Simonetti, todos da Vale, pela valiosa contribuição nas atividades de amostragem e beneficiamento, bem como no intercâmbio de informações e experi- duto final não tem a qualidade exigida pelo mercado. Os altos teores de $\mathrm{Fe}_{2} \mathrm{O}_{3}$ e $\mathrm{TiO}_{2}$ no desareado não diminuem no produto final. Em especial, as amostras desse grupo apresentam uma textura que identifica maior presença de areia fina (quartzo), quando comparadas com as amostras dos demais grupos. A alta quantidade de quartzo inicial no ROM e a persistência dos altos teores de ferro e titânio no produto final indicam que essa variedade de caulim não deve ser beneficiada com os atuais processos utilizados na Mina Ipixuna.

das com as comentadas nos demais grupos, fariam com que o caulim do Grupo VI fosse descartado para o beneficiamento, pelo critério do tipo visual. No entanto, seu produto final tem elevada alvura, compatível com a alvura do produto final do Grupo II; e com as demais características muito próximas com as do produto final do Grupo I, cujo ROM gera produto final de melhor qualidade. Nesse contexto, o critério da variedade espectrocolorimétrica é decisivo para o aproveitamento econômico do minério ROM classificado como pertencente ao Grupo VI, o qual deve ser beneficiado sem blendagem.

As diferenças entre os valores dos parâmetros na Tabela 1 , bem como as evoluções das curvas com os processos, mostradas nas Figuras 2 a 9, sugerem que a paragênese mineral dos contaminantes seja diversa nos diferentes grupos, notadamente quanto aos minerais de ferro. O processo de branqueamento é mais eficaz em algumas amostras e menos em outras. Isso pode ser observado nos teores de $\mathrm{Fe}_{2} \mathrm{O}_{3}$ dos Grupos VI e VII, por exemplo. E muito provável que seja esta a explicação para as diferentes eficácias no processo de branqueamento dos grupos. Estudos sobre a paragênese mineral no ROM e nos rejeitos da centrifugação e da separação magnética estão em andamento, para se entenderem melhor as performances no beneficiamento.

ências profissionais durante os estudos.

Agradecimentos também ao CNPq, pelos recursos financeiros de uma bolsa PQ ao primeiro autor, para o desenvolvimento dos estudos apresentados. 


\section{Referências bibliográficas}

BLOODWORTH, A. J., HIGHLEY, D. E., MITCHELL, C. J. Industrial Minerals Laboratory Manual: Kaolin. British Geological Survey Technical Report WG/93/1, Mineralogy and Petrology Series, Nottingham, United Kingdom, 1993, 80 p.

LUZ, A. B., CAMPOS, A. R., CARVALHO, E. A., BERTOLINO, L. C. Caulim. In: Rochas \& Minerais Industriais: usos e especificações. LUZ, A.B., LINS, F.A.F. (Ed.). CETEM/MCT. Rio de Janeiro: 2005. p. 231-262.

MURRAY, H. H. Traditional and new applications for kaolin, smectite and palygorskite: a general overview. Applied Clay Science, n. 17, p. 207-221, 2000.

PRASADA, M. S., REID, K. J., MURRAY, H. H. Kaolin: processing, properties and application. Applied Clay Science, Amsterdam, v.6, pp.87-119, Elsevier, processing and markets. Mining Engineering, p.79-84, 1991.

ROSKILL. The economics of kaolin. Twelfth Edition. 2006, 4p. In: www.roskill.com. SABEDOT, S., PETTER, C. O., CORREIA, A. G. Procedimentos para a caracterização de caulins por espectro-colorimetria. In: ENCONTRO NACIONAL DE TRATAMENTO DE MINÉRIOS E METALURGIA EXTRATIVA, 17 e SEMINÁRIO DE QUÍMICA DE COLÓIDES APLICADA À TECNOLOGIA MINERAL, 1. Águas de São Pedro, SP: Édile Serviços Gráficos e Editora Ltda., v. 1. p. 449-468, 1998.

SENA, G. L. S., MÁRTIRES, R. A. C. Caulim. In: Sumário Mineral, Departamento Nacional de Produção Mineral, Brasília, DF, 2008.

Artigo recebido em 11 de junho de 2010. Aprovado em 12 de julho de 2011. 